\title{
Segmentación fonológico-silábica y adquisición de la lectura: un estudio empírico
}

\section{María Soledad Carrillo y Julio Sánchez}

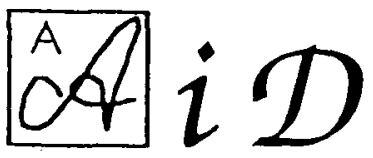

El artículo presenta una investigación cuyos resultados muestran la menor capacidad de segmentación silábica entre aquellos niños con peor babilidad lectora. Plantea también la funcionalidad, facilidad de aplicación y carácter motivador de la prueba realizada - una tarea de ordenador-para evaluar la capacidad de segmentación.

\section{INTRODUCCION}

La capacidad de segmentación fonológico-silábica de las palabras es considerada como uno de los mejores predictores del nivel lector (Liberman y Shankweiler, 1985; Morais, 1987; Stanovich, 1986; Wagner y Torgesen, 1987). Son numerosísimas las investigaciones que en la última década se han ocupado en determinar la importancia que para la lectura de lenguas alfabéticas tiene la conciencia de los sonidos del lenguaje oral y la segmentación en unidades fonémicas, silábicas o suprasilábicas (Bradley y Bryant, 1983, 1985; Calfee, Lindamood y Lindamood, 1973; Fox y Routh, 1975; Liberman, Shankweiler, Fischer y Carter, 1974; Lundberg, Olofsson y Walls, 1980; Share, Jorm, McLean y Matthews, 1984; Treiman y Baron, 1981, 1983).

Dentro de esta línea de investigación se admite con toda rotundidad que las habilidades de segmentación juegan un papel fundamental en las etapas iniciales del aprendizaje de la lectura, ya que no sólo permiten al niño comprender las relaciones entre el texto escrito y su propio lenguaje (Alegría y Laybaert, 1987), sino que además facilitan el proceso de decodificación tan arduo en sus comienzos.

Las habilidades de segmentación fonológica de más alto nivel - -segmentación fonémica - no surgen en la mayoría de los casos sin la instrucción más o menos explícita que, de alguna manera, acompaña a los sistemas de enseñanza de la lectura de lenguas alfabéticas (Morais, Alegría y Content, 
1987). Pero otras formas de análisis fonológico, como son la detección y producción de la rima y la aliteración, o la capacidad de segmentación silábica, preceden al contacto con el material escrito (Bradley, 1988; Cossu, Shankweiler, Liberman, Katz y Tola, 1988; Maldonado y Sebastián, 1987).

La utilidad que para el aprendizaje de la lectura pueda tener cada uno de estos tipos de segmentación podría depender de algunas características concretas de cada lengua alfabética en particular, como pueden ser el número y complejidad de tipos distintos de sílabas y letras y la regularidad de sus traducción fonológica.

En el castellano, la sílaba constituye una unidad de articulación fácilmente aislable de la cadena hablada, existiendo una regularidad casi perfecta en la correspondencia entre formas escritas y fonológicas. Por todo ello, el proceso de enseñanza de la lectura en nuestra lengua podría descansar en sus momentos iniciales en el establecimiento de correspondencias entre las unidades silábicas escritas y sus correspondientes unidades de articulación.

Los intentos por determinar las causas del retraso específico en lectura, así como los realizados por establecer prerrequisitos o condiciones óptimas para aprender a leer no siempre se han dirigido hacia capacidades o conductas relacionadas inequívoca o específicamente con la actividad lectora (Maldonado - Sebastián, 1987). Por consiguiente, el adiestramiento o la madurez en algunos supuestos «prerrequisitos» no han venido acompañados de un éxito o mejora en la lectura.

En resumen, nuestro planteamiento parte de dos consideraciones básicas. En primer lugar, que las capacidades de segmentación fonológica son un componente fundamental de la habilidad lectora en lenguas de ortografía alfabética; $y$, en segundo lugar, que en la lengua castellana los segmentos silábicos constituyen unidades de especial relevancia en el proceso de adquisición de la lectura.

En consecuencia, el objetivo del presente trabajo tiene una finalidad tanto teórica como aplicada. Desde un punto de vista teórico, se pretende demostrar la relación existente entre la capacidad de segmentación fonológico-silábica de palabras y el nivel lector. Desde su vertiente aplicada, se pretendió diseñar un instrumento informatizado de fácil aplicación en el marco escolar a niños de temprana edad, que permitiera evaluar la habilidad de segmentación fonológica, así como la detección temprana de posibles problemas para el posterior aprendizaje de la lectura.

\section{METODO}

\section{Sujetos}

Los niños que participaron en la experiencia fueron seleccionados del colegio público Santa M. ${ }^{a}$ de Gracia situado en el casco urbano de Murcia capital, siendo la extracción social de nivel medio. Para la obtención de las dos muestras de niños, una de lectores normales (BL) y otra de niños con «retraso específico en lectura» (ML), se aplicó el T.A.L.E. (Test de Análisis de Lecto-Escritura; Cervera y Toro, 1980) y se combinó esta información con el criterio del maestro para asignar a los niños a uno u otro grupo de 
habilidad lectora. De esta forma, se seleccionaron 40 niños agrupados de la siguiente forma: 10 niños $\mathrm{BL}$ ( 5 niños y 5 niñas) y 10 niños ML (8 niños y 2 niñas) de $1 .^{\circ}$ de E.G.B.; y 10 niños BL ( 5 niños y 5 niñas) y 10 niños ML (7 niños y 3 niñas) de $2 .^{\circ}$ de E.G.B. Se controló la inteligencia aplicando la versión castellana del Raven. En la Tabla I se presentan los percentiles medios en inteligencia, así como las edades de cada grupo de niños. Mediante análisis de varianza se comprobó que los dos grupos de habilidad lectora no diferían en inteligencia, $\mathrm{F}(1,36)<1$, ni en edad, $\mathrm{F}(1,36)=2,937, \mathrm{p}=.095$. Además, el nivel de inteligencia fue similar para los dos cursos y no se observó interacción entre el nivel lector y el curso; tampoco resultó significativa esta interacción en cuanto a las edades, $\mathrm{F}(1,36)<1$ en todos los casos.

\section{TABLA I}

Edades y niveles de inteligencia medios registrados en cada grupo de sujetos según el nivel lector $(B L$ vs. $M L)$ y el curso $\left(10^{\circ}\right.$ vs. $2^{\circ}$ de E.G.B.). El nivel de inteligencia se presenta en percentiles. Los valores entre paréntesis son las derivaciones típicas.

\begin{tabular}{|c|c|c|c|c|c|c|}
\hline \multirow{3}{*}{$\begin{array}{l}\text { NIVEL } \\
\text { LECTOR }\end{array}$} & \multicolumn{4}{|c|}{ CURSO } & & \\
\hline & \multicolumn{2}{|c|}{$1 .^{\circ}$} & \multicolumn{2}{|c|}{$2 .^{\circ}$} & \multicolumn{2}{|c|}{ TOTALES } \\
\hline & Edad & Intelig. & Edad & Intelig. & Edad & Intelig. \\
\hline $\begin{array}{l}\text { Buenos } \\
\text { Lectores }\end{array}$ & $\begin{array}{c}6,758 \\
(0,549)\end{array}$ & $\begin{array}{c}85,50 \\
(11,65)\end{array}$ & $\begin{array}{c}7,892 \\
(0,381)\end{array}$ & $\begin{array}{c}79,50 \\
(15,36)\end{array}$ & $\begin{array}{c}7,325 \\
(0,741)\end{array}$ & $\begin{array}{c}82,500 \\
(13,621)\end{array}$ \\
\hline $\begin{array}{l}\text { Malos } \\
\text { Lectores }\end{array}$ & $\begin{array}{c}6,567 \\
(0,466)\end{array}$ & $\begin{array}{c}85,50 \\
(10,12)\end{array}$ & $\begin{array}{c}7,558 \\
(0,524)\end{array}$ & $\begin{array}{c}85,20 \\
(7,254)\end{array}$ & $\begin{array}{c}7,063 \\
(0,701)\end{array}$ & $\begin{array}{l}85,350 \\
(8,573)\end{array}$ \\
\hline TOTALES & $\begin{array}{c}6,663 \\
(0,505)\end{array}$ & $\begin{array}{c}85,50 \\
(10,62)\end{array}$ & $\begin{array}{c}7,725 \\
(0,478)\end{array}$ & $\begin{array}{c}82,35 \\
(12,05)\end{array}$ & & \\
\hline
\end{tabular}

Estos análisis nos permitieron comprobar que los grupos de niños estaban igualados en inteligencia y edad. Por otra parte, la selección de los sujetos incluyó otros criterios, tales como la ausencia de problemas de conducta, emocionales o sensoriales, y llevar un rendimiento escolar normal y asistencia regular a las clases. Además, los niños $\mathrm{ML}$ debían exhibir un retraso en su rendimiento lector según su edad y curso.

\section{Instrumentos}

Para la realización de la experiencia, se dispuso de la microaula del colegio, dotada con ordenadores IBM PS/2. La tarea de segmentación fonológicosilábica fue programada en GWBasic. El programa permitió presentar en la pantalla del ordenador dibujos familiares a los niños, pero con diferente nivel de dificultad, según el número y ripo de sílabas de los nombres. En la Tabla II se presentan los nombres de los 30 dibujos utilizados, de los cuales 10 correspondían a palabras bisílabas, 10 a palabras trisílabas y los 10 restantes a palabras tetrasílabas. 
TABLA II

Relación de los nombres correspondientes a los dibujos utilizados en la tarea de segmentación fonológico-silábica.

\begin{tabular}{|l|l|l|}
\hline \multicolumn{1}{|c|}{ Bisílabas } & \multicolumn{1}{|c|}{ Trisílabas } & \multicolumn{1}{c|}{ Tetrasílabas } \\
\hline PAVO & CORAZÓN & ZAPATILLAS \\
PASTEL & HELADO & TELEVISOR \\
BEBÉ & CABALLO & ORDENADOR \\
CESTA & PINGÜINO & CAMPANILLAS \\
COCHE & CARACOL & CALABAZA \\
BARCO & PAJARO & CALAVERA \\
GATO & GUITARRA & HAMBURGUESA \\
LIBRO & PAYASO & DINOSAURIO \\
ARBOL & BOMBILLA & TELEFONO \\
CASA & ESTRELLA & CANDELABRO \\
\hline
\end{tabular}

\section{Procedimiento}

La aplicación de la tarea fue individual y siempre en horas escolares. En primer lugar, el niño se sentaba ante el ordenador y se les explicaban las instrucciones. A continuación, se le administraban algunos ensayos de práctica y finalmente tenía lugar la fase experimental propiamente dicha compuesta por 30 ensayos correspondientes a los 30 dibujos de la Tabla II. La duración total de la experiencia con cada niño fue de unos 5 minutos.

Cada ensayo experimental constaba de los siguientes pasos. En primer lugar, aparecía en la pantalla del ordenador un dibujo con su nombre; seguidamente, el monitor pronunciaba en voz alta la palabra y, a continuación el niño debía presionar la barra espaciadora del teclado del ordenador tantas veces como sílabas tuviese la palabra. El programa computaba la precisión de las respuestas grabando las respuestas correctas, los errores por defecto (el número de presiones de la barra era inferior al correcto) y los errores por exceso (el número de presiones era superior). Además, el programa incluye una rutina que permitió aleatorizar el orden de presentación de los 30 dibujos para cada niño.

\section{Diseño}

Para el análisis de los resultados se definieron tres índices de precisión sobre el total de los 30 ensayos: El número de respuestas correctas, el número de errores por defecto y el número de errores por exceso. Sobre cada índice de precisión se aplicó un análisis de varianza factorial con dos factores: el nivel lector (BL vs. ML) y el curso (1. ${ }^{\circ}$ vs. $2 .^{\circ}$ de E.G.B.). Todos los análisis se realizaron con el paquete estadístico SYSTAT (Wilkinson, 1988).

\section{RESULTADOS}

Las Tablas III(a), (b) y (c) presentan los porcentajes medios de respuestas 
correctas, errores por defecto y errores por exceso para cada grupo de niños según el nivel lector y el curso. Globalmente, los resultados indican que la tarea resultó bastante sencilla para los niños de estos niveles de edad, ya que hubo un $92,10 \%$ de respuestas correctas. Pero el resultado más interesante es la evidencia de una mejor capacidad de segmentación de palabras en los niños BL $(94,66 \%$ de aciertos) que en los ML (89,54\%), según se revela del análisis de varianza efectuado sobre el número de respuestas correctas, $F(1,36)=5,054, p=.031$. No se observaron diferencias significativas debidas al curso ni a su interacción con el nivel lector, $F(1,36)<1$.

TABI.A III

Porcentajes medios obtenidos en la tarea de segmentación fonológico-silábica por cada grupo de niños.

(a) RESPUESTAS CORRECTAS (\%)

\begin{tabular}{|c|c|c|c|c|}
\hline \multirow{5}{*}{$\begin{array}{l}\text { NIVEL } \\
\text { LECTOR }\end{array}$} & \multicolumn{4}{|c|}{ CURSO } \\
\hline & & $1 .^{\circ}$ & 2. ${ }^{\circ}$ & \multirow{3}{*}{$\begin{array}{l}94,66 \\
89,54\end{array}$} \\
\hline & $\mathrm{BL}$ & 93,33 & 96,00 & \\
\hline & ML & 88,75 & 90,33 & \\
\hline & \multicolumn{2}{|r|}{91,04} & 93,16 & 92,10 \\
\hline
\end{tabular}

(b) ERRORES POR DEFECTO (\%)

\begin{tabular}{|c|c|c|c|c|}
\hline \multirow{5}{*}{$\begin{array}{l}\text { NIVEL } \\
\text { LECTOR }\end{array}$} & \multirow{4}{*}{$\begin{array}{l}\text { BL } \\
M L\end{array}$} & \multicolumn{2}{|c|}{ CURSO } & \multirow[b]{3}{*}{0,35} \\
\hline & & $1 .^{\circ}$ & $2 .^{\circ}$ & \\
\hline & & 0,37 & 0,33 & \\
\hline & & 2,50 & 5,00 & 3,75 \\
\hline & & 1,435 & 2,67 & 2,05 \\
\hline
\end{tabular}

(c) ERRORES POR EXCESO (\%)

\begin{tabular}{|c|c|c|c|c|}
\hline \multirow{5}{*}{$\begin{array}{l}\text { NIVEL } \\
\text { LECTOR }\end{array}$} & \multirow{4}{*}{$\begin{array}{l}\text { BL } \\
\text { ML }\end{array}$} & \multicolumn{2}{|c|}{ CURSO } & \multirow{4}{*}{$\begin{array}{r}4,985 \\
6,71\end{array}$} \\
\hline & & $1 .^{\circ}$ & $2 .^{\circ}$ & \\
\hline & & 6,30 & 3,67 & \\
\hline & & 8,75 & 4,67 & \\
\hline & \multicolumn{2}{|r|}{7,525} & 4,17 & 5,85 \\
\hline
\end{tabular}

La Figura 1 permite examinar con mayor claridad la precisión de los niños en la tarea según el curso y el nivel de habilidad lectora. Se observa cómo las diferencias en ejecución entre BL y ML se mantiene constante a través de los dos cursos. Es decir, los niños BL reflejaron una mejor capacidad de segmentación de sílabas que los ML tanto en $1 .^{\circ}$ como en $2 .^{\circ}$ de E.G.B. Este 
resultado no contradice la hipótesis formulada por numerosos autores de que la capacidad de análisis fonológico comienza a desarrollarse antes del inicio del aprendizaje de la lectura, pero que posteriormente, ambas habilidades - leer y segmentar palabras-, se complementan mutuamente (Cataldo y Ellis, 1988; Ellis y Large, 1987, 1988; Wagner y Torgesen, 1987).

\section{Figura 1}

Número medio de respuestas correctas obtenidas en la tarea de segmentación según el nivel de habilidad lectora ( $B L$ vs. $M L$ ) y el curso.

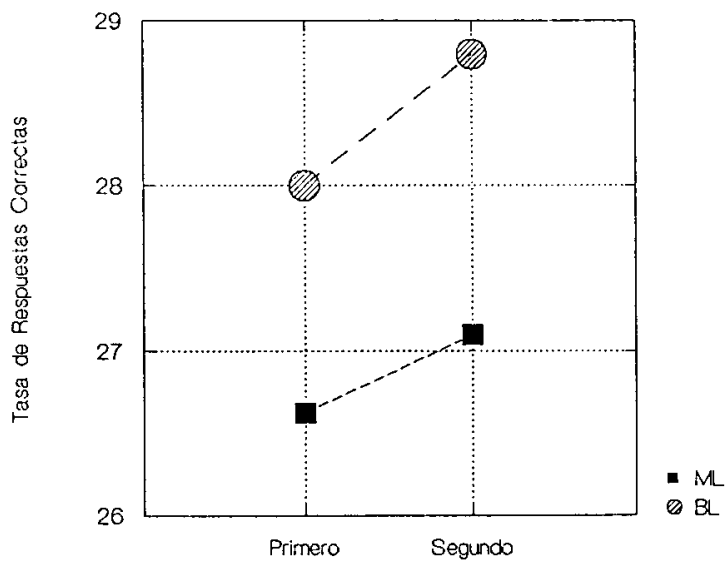

El análisis de los errores demostró que, globalmente, los niños cometieron casi el triple de errores por exceso $(5,85 \%)$ que por defecto $(2,05 \%)$, $t(39)=2,724, p=.010$; posiblemente debido a la presencia de diptongos en algunas de las palabras utilizadas en la experiencia. Pero no se observaron diferencias significativas entre BL y ML en cuanto a la tasa de errores por defecto, $\mathrm{F}(1,36)=2,639, \mathrm{p}=.113$; ni en cuanto a la tasa de errores por exceso, $F(1,36)=1,462, p=.234$. Por tanto, ambos grupos de niños manifestaron la misma tendencia a cometer menos errores por defecto que por exceso (véanse las Tablas III (b) y (c)). Sin embargo, se observó un descenso en la tasa de errores por exceso con el curso $(7,525 \%$ vs. $4,17 \%), F(1,36)=5,525, p=.024$.

\section{CONCLUSIONES}

Resumiendo, pues, podemos decir que, a pesar de la facilidad con que ejecutaron globalmente la tarea, los niños ML presentaron una capacidad de segmentación silábica significativamente inferior a la mostrada por los lectores normales de su misma edad. En consecuencia, nuestros resultados confirman la relación existente entre la adquisición de la lectura y la habilidad de segmentación de palabras.

Sin embargo, esta relación no parece suficiente para explicar un alto porcentaje de la variabilidad en la competencia lectora de los niños. Esto puede explicarse por el tipo de tarea diseñada, que podría no reflejar una representación consciente del lenguaje en sus unidades silábicas, sino más bien un tipo de habilidad que opera a nivel de «simple percepción del habla». Pensamos que otras tareas más complejas de manejo de unidades silábicas, tales como inversión, omisión, adición, etc., serían capaces de reflejar un nivel de procesamiento postperceptivo más fuertemente predictor de la habilidad lectora (Morais et al., 1987). 
Una cuestión crucial y de difícil resolución en el actual estado de la investigación en el ámbito internacional es el referido a la dirección causal de las relaciones encontradas entre rendimiento lector y habilidades de análisis fonológico. La investigación que aquí presentamos no permite avanzar en la resolución de este aspecto. No obstante, recientes investigaciones con niños de habla inglesa (Cataldo y Ellis, 1988; Ellis y Large, 1988) han permitido constatar, mediante la aplicación de modelos causales, la presencia de habilidades fonológicas en niños prelectores, así como la bidireccionalidad de la relación entre estos dos constructos psicológicos una vez iniciado el aprendizaje de la lectura.

Por otra parte, el medio utilizado para la presentación de los estímulos y la recogida de respuestas, el ordenador, tiene sin duda amplias ventajas sobre la aplicación tradicional de estas tareas. Entre tales ventajas destacamos, con carácter general, su valor motivador para el niño, que encara la situación de evaluación como un juego; además este medio es capaz de atraer y mantener mejor la atención del niño, lo que es de gran interés para que la ejecución de la tarea refleje sus capacidades. A estas ventajas se añaden las derivadas de la uniformidad en la presentación de los estímulos, tiempos de exposición, secuenciación de los ensayos, etc. Entre las ventajas de carácter particular, el uso del ordenador facilita la recogida de las respuestas de precisión y de tiempo de ejecución, así como la aleatorización automática del orden de presentación de los estímulos. Asímismo, como instrumento de apoyo para la realización de investigaciones, el ordenador nos ha facilitado la tabulación directa de los datos, con todas las posibilidades que la programación informática ofrece.

Resumiendo, consideramos que una tarea de segmentación fonológicosilábica como la que presentamos en este trabajo resulta un instrumento de gran utilidad en el aula para la evaluación del nivel de habilidades de segmentación fonológico-silábica en niños que se están iniciando en el aprendizaje de la lectura, así como un complemento para la predicción de problemas en dicho aprendizaje.

\section{Referencias}

Aili:GRIA, J. y LI:YBARR', J. (1987). Adquisición de la lectura en el niño sordo. Madrid: M.E.C., Dirección General de Renovación Pedagógica.

Bramili, L. (1988). Making comnections in learning to read and to spell. Applied Cognitive Psychology, 2, 3-18.

Bradils, L. y BRrant, P. E. (1983). Categorizing sounds and learning to read: A causal connection. Nature, 301, 419-421.

Bramil:y, L. y Bryant, P. E. (1985). Rhyme and Reason in Reading and Spelling. Ann Arbor, MI: University of Michigan State.

Calliti, R.; Linisamoon, P. y Lindamood, C. (1973). Acousticphonetic skills in reading -kindergarten through twelfth grade. Journal of Educational Psychology, 64, 293-298.

CATAIDO, S. y El.I.IS, N. (1988). Interactions in the development of spelling, reading, and phonological skills. Journal of Research in Reading, I/(2), 86-109.

Cirvi:ka, M. y Toro, J. (1980). T.A.L.E.: Test de Análisis de la Lecto-Escritura. Madrid: Visor Libros.

Cossu, G.; Shankwillitr, D.; Libi:kman, I. Y.; KaT\%, L. y Tol.A, G. (1988). Awareness of phonological segments and reading ability in italian children. Applied Psycholinguistics, 9, $1-16$.

Ei.IIs, N. y Lakcil, B. (1987). The development of reading: As you seek so shall you find. British Journal of Psychology, 78, 1-28.

El.I.1s, N. y LAKGE, B. (1988). The carly stages of reading: A longitudinal study. Applied Cognitive Pychology, 2, 47-76. 
Fi)x, B. y Routh, D. (1975). Analyzing spoken language into words, syllabes, and phonemes: A developmental study. Journal of Psycholinguistic Research, 4, 331-342.

Libi:Rman, I. y ShankwiniliR, D. (1985). Phonology and the problems of learning to read and write. Remedial y Special Education, 6, 8-17.

Libi:Rman, I.; Shankwillik, D.; Fischi:r, F. y Cartek, B. (1974). Explicit syllabe and phoneme segmentation in the young child. Journal of Experimental Child Psychology, 18, $201-212$.

LUNIIII:RC; I.; Oi.OISSON, A. y WALLS, S. (1980). Reading and spelling skills in the first school years predicted from phonemic awareness skills in kindergarten. Scandinavian Journal of Psychology, 21, 159-173.

Mai.iponaido y Shibastian, E. (1987). La segmentación de palabras: Un prerrequisito del aprendizaje de la lectura. Boletín I.C.E. (U.A.M.), 9 (enero), 5-14.

Morais, J. (1987). Phonetic awareness and reading acquisition. Psycbological Research, 49, 147152.

Morais, J.; Aifigria, J. y Content, A. (1987). The relationship between segmental analysis and alphabetic literacy: An interactive view. Cabiers de Psychologie Cognitive, 7, 415-438.

Shari, D.; Jorm, A.; Mcilian, R. y MatTHEws, R. (1984). Sources of individual differences in reading acquisition. Journal of Educational Psychology, 76, 1309-1324.

SIANOVICH, K. (1986). Matthew effects in reading: Some consequences of individual differences in the acquisition of literacy. Reading Research Quarterly, 21(4), 360-407.

Tri:lman, R. y Baron, J. (1981). Segmental-analysis ability: Development and relation to reading ability. En G. McKinnon y T. Waller (Eds.), Reading Research: Advances in Theory and Practice, vol. 3. Nueva York: Academic Press.

Tri:iman, R. y BARON, J. (1983). Phonemic-analysis training helps children benefit from spelling-sound rules. Memory y Cognition, 11, 382-389.

WAGNeR, R. K. y TORGESIN, J. K. (1987). The nature of phonological processing and its causal role in the acquisition of reading skills. Psychological Bulletin, 101(2), 192-212.

WII.KInson, L. (1988). SYSTAT: The System for Statistics (Vers. 4.0). Evanston, IL: SYSTAT, Inc.

\section{Segmentación fonológico-silábica y adquisición de la lectura: Un estudio empírico. M. S. Carrillo y J. Sánchez. CLEE, 1991, 9, pp. 109-116}

Resumen: Con objeto de estudiar la relación existente entre babilidad lectora y capacidad de segmentación de palabras, se seleccionó una muestra de 40 niños de $10^{\circ}$ y $2 .^{\circ}$ de E.G.B., clasificados según su nivel de lectura (buenos vs. malos lectores) y se les aplicó una tarea de segmentación fonológico-silábica presentada por ordenador. Los resultados muestran la existencia de una mayor capacidad de segmentación de silabas en los lectores normales que en los niños con retraso especifico en lectura, tanto en $10^{\circ}$ como en $2^{\circ}$ de E.G.B. Además, la simplicidad de la tarea implementada la convierte en un instrumento muy útil en el marco escolar para la detección temprana de niños con posibles problemas en el aprendizaje de la lectura.

Datos sobre los autores: María Soledad Carrillo Gallego imparte Psicología Evolutiva de la Edad Preescolar en la Escuela de Formación del Profesorado de E.G.B. de Murcia. Sus intereses de investigación se centran en las etapas iniciales del aprendizaje de la lectura. Julio Sánchez Meca imparte Metodología de la Investigación en Ciencias del Comportamiento en la Facultad de Filosofía, Psicología y Ciencias de la Educación de Murcia. Sus intereses de investigación se centran en los métodos de investigación en psicología de la lectura.

Dirección: M. S. Carrillo, Universidad de Murcia, Departamento de Psicología Evolutiva y de la Educación, Campus de Espinardo, 30071 Murcia.

Agradecimientos: Este trabajo ha sido subvencionado por una Beca de la Comisión Asesora de Investigación Científica y Técnica, CAICYT, bajo la dirección del Dr. Manuel Ato García (referencia: PA85-0258). Parte de estos resultados se presentaron al $I^{\circ}$ Symposium Nacional de Metodología de las Ciencias Humanas, Sociales y de la Salud, Salamanca, noviembre de 1989.

๑ de todos los artículos. Deberá solicitarse por escrito autorización de CL\&E para el uso en forma de facsímil, fotocopia o cualquier otro medio de reproducción. CL\&E se reserva el derecho de interponer las acciones legales necesarias en aquellos casos en que se contravenga la ley de derechos de autor. 